\title{
Incoherent scatter radar plasma density measurements at Jicamarca using a transverse-mode differential-phase method
}

\author{
Erhan Kudeki, ${ }^{1}$ Ronald F. Woodman, ${ }^{2}$ and Zhaomei Feng ${ }^{1}$ \\ Received 16 May 2002; revised 2 September 2002; accepted 26 December 2002; published 14 March 2003.
}

[1] The $50 \mathrm{MHz}$ Jicamarca incoherent scatter radar can be used to make very high precision F-region plasma drift measurements with less than a $\mathrm{m} / \mathrm{s}$ uncertainty and $5 \mathrm{~min} /$ $15 \mathrm{~km}$ time/height resolutions. In such measurements the transmitting antenna beam is pointed perpendicular to the geomagnetic field $\vec{B}$ [e.g., Kudeki et al., 1999] and backscattered fields consist of magneto-ionic $\mathrm{O}$ - and $\mathrm{X}$ components with unequal phase retardations. Detecting the fields with an orthogonal pair of linear-polarized antennas and fitting the average power and differentialphase of the antenna outputs to an appropriate data model we have succeeded in making F-region electron density measurements with data collected during Jicamarca drifts experiments. This procedure provides Jicamarca with a new capability for simultaneous drifts and density measurements at F-region heights. INDEX TERMS: 6969 Radio Science: Remote sensing; 2415 Ionosphere: Equatorial ionosphere; 6934 Ionospheric propagation (2487); 6994 Instruments and techniques. Citation: Kudeki, E., R. F. Woodman, and Z. Feng, Incoherent scatter radar plasma density measurements at Jicamarca using a transverse-mode differential-phase method, Geophys. Res. Lett., 30(5), 1255, doi:10.1029/2002GL015496, 2003.

\section{Introduction}

[2] A new incoherent scatter radar technique is being developed at Jicamarca for making F-region electron density measurements with antenna beams pointed perpendicular to the geomagnetic field. The project aims to provide density results to accompany the high-precision F-region drifts data collected using the same beam orientations [e.g., Kudeki et al., 1999]. The purpose of this paper is to describe the new technique and present preliminary results obtained so far.

[3] The relative orientations of the geomagnetic field $\vec{B}$ and the radar beam above Jicamarca influence both the Doppler frequency spectrum and propagation phase delay of magneto-ionic subcomponents of backscattered radar fields. When the beam is perpendicular to $\vec{B}$ the fields are transverse propagating linear-polarized magneto-ionic modes [e.g., Ratcliffe, 1962] and have a relatively narrow frequency spectrum [e.g., Kudeki et al., 1999]. But when the radar beam deviates from the transverse direction by as little as a few degrees the frequency spectrum is severely broadened and propagation is in terms of nearly circular polarized quasi-longitudinal modes. At the $50 \mathrm{MHz}$ operation frequency of the Jicamarca radar, the critical angle

\footnotetext{
'Department of Electrical and Computer Engineering, University of Illinois at Urbana-Champaign, Urbana, Illinois, USA.

${ }^{2}$ Instituto Geofisico del Peru, Lima, Peru.
}

Copyright 2003 by the American Geophysical Union. 0094-8276/03/2002GL015496\$05.00 which separates the quasi-longitudinal and -transverse regimes of ionospheric propagation is only about $0.5^{\circ}$ off the direction perpendicular to $\vec{B}$.

[4] Since high-precision drifts measurements favor radar return signals with the narrowest possible frequency spectra, Jicamarca drifts observations are conducted using the linearpolarized transverse radar beams rather than the circularpolarized quasi-longitudinal beams used in Faraday rotation density measurements [Farley, 1969]. To supplement the Jicamarca drifts data with F-region density estimates we now collect the transverse-beam signal returns using an orthogonal pair of linear-polarized antennas and fit the average power and phase difference of the antenna outputs to an appropriate data model described in section 2 . The new procedure is partly an extension of the Faraday rotation method [Farley, 1969] into the magneto-ionic regime of nearly linear polarized quasi-transverse propagation modes and partly a procedure of Valladares and Woodman [2001] based on collecting all components of the backscattered radar signal power. It is also based on the principles of maximum-likelihood data inversion [e.g., Menke, 1989].

[5] Preliminary results obtained with the new density estimation technique will be presented and discussed in section 3 . Since the new technique makes use of a single beam direction it avoids the reduced sensitivity problem of the earlier Jicamarca drifts and density experiments [e.g., Woodman et al., 1972] conducted with multiple beams. Also, as discussed in section 3 , the technique holds the promise to provide electron density estimates within equatorial spread-F layers and bubbles.

\section{Data Acquisition and Inversion}

\subsection{Orthogonal Receiver Signals}

[6] The antenna array at Jicamarca consists of two orthogonal sets of half-wave dipoles with south-east and north-east polarizations, respectively. In reception mode we call the baseband voltage of the sum and difference of the orthogonal array outputs (obtained by using a hybridcombiner prior to coherent detection) $V_{\circ}$ and $V_{\theta}$. The sum $V_{\mathrm{o}}$ is a complex voltage that samples the zonal component of the backscattered electric field. The difference $V_{\theta}$ samples the meridional component.

[7] When Jicamarca antenna beams are steered to point perpendicular to the geomagnetic field $\vec{B}$, the radar output voltages $V_{\theta}$ and $V_{o}$ correspond to respective samples of linear-polarized ordinary- and extraordinary-mode radar returns parallel and perpendicular to $\vec{B}$. The differential phase (in radians) of $V_{\theta}$ and $V_{\circ}$ is then

$$
\angle V_{\theta} V_{\dot{\phi}}^{*}=-2 k_{o} \int_{0}^{z} d z^{\prime} \delta n\left(z^{\prime}\right) \approx-\frac{80.6 \Omega^{2}}{c f^{2} \omega} \int_{0}^{z} d z^{\prime} N\left(z^{\prime}\right),
$$


where $\delta n \equiv n_{o}-n_{x}$ is the difference in ordinary- and extraordinary-mode refractive indices $n_{o}$ and $n_{x}, 80.6 \approx \frac{e^{2}}{m_{e} \epsilon_{o}}$ in MKS units, $\Omega$ the electron gyro-frequency, $k_{o} \equiv \frac{\omega}{c}, \stackrel{\omega}{\omega}=$ $2 \pi f$ the radar frequency, $z$ the scattering height, and $N(z)$ the ionospheric electron density profile. Expression (1) differs from the differential-phase of the right- and leftcircular modes used in Faraday rotation method [Farley, 1969 ] by only a multiplicative factor of $\frac{\Omega / \omega}{2 \cos \theta_{o}} \approx 0.15$, where $\theta_{o} \approx 87^{\circ}$ is the Jicamarca beam pointing angle in the Faraday mode away from $-\vec{B}$. Expression (1) therefore suggests the possibility of inferring the ionospheric density profile $N(z)$ from the differential phase of the orthogonal components of transverse-beam radar returns.

[8] However, since the Jicamarca beams have a finite angular width and because the magnetic field vector $\vec{B}$ varies with altitude, the scattered signals are not strictly linear polarized and propagate in mixture with elliptic polarized quasi-transverse $\mathrm{O}$ - and $\mathrm{X}$-modes. As a consequence, the inversion process used to estimate $N(z)$ from the transverse beam radar data requires a more accurate differential-phase model than just (1). The inversion process described next also makes use of backscattered power data $P_{\theta}$ and $P_{\mathrm{o}}$ measured in $\theta$ - and $\phi$-channels, respectively.

\subsection{Data Models and Data/Model Misfit}

[9] Let $P_{\theta}$ and $P_{\mathrm{o}}$ denote the measured average power in $\theta$ - and $\phi$-channels of a transverse-beam incoherent scatter radar experiment outlined above. Ideally then, $\left\langle P_{\theta, o}\right\rangle=$ $\left\langle\left|V_{\theta, o}\right|^{2}\right\rangle$-where $\langle\cdot\rangle$ denotes expected value-but in practice $\left\langle P_{\theta}\right\rangle=\left\langle\left|V_{\theta}\right|^{2}\right\rangle+A_{\theta}$ and $\left\langle P_{\mathrm{o}}\right\rangle=|g|^{2}\left\langle\left|V_{\mathrm{o}}\right|^{2}\right\rangle+A_{\mathrm{O}}$ because of additive noise power $A_{\theta, \mathrm{O}}$ in reception channels and relative voltage gain $g$ of $\phi$-channel receiver over the $\theta$ channel receiver. Thus,

$$
P \equiv|g|^{2} P_{\theta}+P_{\circ}=|g|^{2}\left(\left\langle\left|V_{\theta}\right|^{2}\right\rangle+\left\langle\left|V_{\circ}\right|^{2}\right\rangle\right)+|g|^{2} A_{\theta}+A_{\circ}+\delta P,
$$

where $\delta P$ is a zero-mean random fluctuation in $|g|{ }^{2} P_{\theta}+P_{\mathrm{c}}$ about its expected value $|g|^{2}\left\langle P_{\theta}\right\rangle+\left\langle P_{\circ}\right\rangle$.

[10] Likewise, let $\psi$ denote the phase angle of the estimate of the complex cross-correlation of $\theta$ - and $\phi$-channel signals. Although $\langle\psi\rangle=\left\langle\left\langle V_{\theta} V_{\circ}^{*}\right\rangle\right.$ ideally, in practice

$$
\psi=\left\langle\left\langle V_{\theta} V_{0}^{*}\right\rangle-\langle g+\delta \psi\right.
$$

because of the phase $\angle g$ of relative gain $g$ as well as random fluctuations $\delta \psi$.

[11] Relations (2) and (3) apply at every data range gate $z_{m}$ where the power and phase data will be labeled as $P_{\theta m}, P_{\mathrm{o} m}$, $\psi_{m}$ and the corresponding data models as $\left\langle\left|V_{\theta}\right|^{2}\right\rangle_{m}+\left\langle\left|V_{\mathrm{o}}\right|^{2}\right\rangle_{m}$, $\left\langle\left\langle V_{\theta} V_{\circ}^{*}\right\rangle_{m}\right.$. With sufficiently long integration times used to compute $P_{\mathrm{t}, \mathrm{om}}, \psi_{m}$, the corresponding fluctuations

$$
\delta P_{m} \equiv|g|^{2}\left(P_{\theta m}-A_{\theta}\right)+\left(P_{\circ m}-A_{\circ}\right)-|g|^{2}\left(\left\langle\left|V_{\theta}\right|^{2}\right\rangle_{m}+\left\langle\left|V_{\circ}\right|^{2}\right\rangle_{m}\right)
$$

and

$$
\delta \psi_{m} \equiv \psi_{m}+\left\langle g-\left\langle\left\langle V_{\theta} V_{\circ}^{*}\right\rangle_{m}\right.\right.
$$

are known to be independent zero-mean Gaussian random variables with a joint $\mathrm{pdf} \propto e^{-\chi^{2} / 2}$, where

$$
\chi^{2} \equiv \sum_{m} \frac{\delta P_{m}^{2}}{\left\langle\delta P_{m}^{2}\right\rangle}+\sum_{m} \frac{\delta \psi_{m}^{2}}{\left\langle\delta \psi_{m}^{2}\right\rangle} .
$$

Furthermore, since $x^{2}$ depends on ionospheric electron densities via $\left\langle\left|V_{\theta}\right|^{2}\right\rangle_{m}+\left\langle\left|V_{\mathrm{o}}\right|^{2}\right\rangle_{m}$ and $\left\langle\left\langle V_{\theta} V_{\circ}^{*}\right\rangle_{m}\right.$ in (4) and (5), the distribution $\propto e^{-\chi^{2}} / 2$ also represents the joint likelihood of electron densities at sampling heights conditioned by ionospheric data $P_{\text {t.om }}, \psi_{m}$. As a consequence we can determine the maximum-likelihood estimates of the densities by minimizing (6) over its free parameters. This procedure can be viewed as finding the best fit of the backscatter radar data to data models $\left\langle\left|V_{\theta}\right|^{2}\right\rangle_{m}+\left\langle\left|V_{\mathrm{o}}\right|^{2}\right\rangle_{m}$ and $\left\langle\left\langle V_{\theta} V_{\circ}^{*}\right\rangle_{m}\right.$ described next.

\subsubsection{Power Model}

[12] For matched-filtered reception of the echoes of uncoded radar pulses of some length $\Delta z$,

$$
\left\langle\left|V_{\theta}\right|^{2}\right\rangle_{m}+\left\langle\left|V_{\odot}\right|^{2}\right\rangle_{m}=\frac{K}{z_{m}^{2}} \frac{N_{m}}{1+\mu_{m}} \equiv \Pi_{m},
$$

where $K$ is a system constant (that depends on transmitted power, antenna aperture, $\mathrm{Ohmic}$ losses, pulse length $\Delta z$, etc.), $N_{m}$ denotes the average electron density between the heights $z_{m-1}=z_{m}-\Delta z$ and $z_{m+1}=z_{m}+\Delta z$, and $\mu_{m}$ is a variable of order unity that depends on the electron-ion temperature ratio $T_{e} / T_{i}$ within the same height range. Model $\Pi_{m}$ is a consequence of incoherent scatter radar cross-sections $\propto N(z)(1+\mu(z))^{-1}$ [e.g., Farley, 1971] and the principle of energy conservation (ignoring the differential absorption of $\mathrm{O}$ - and X-modes which is negligible at $50 \mathrm{MHz}$ ), and is valid independent of the antenna polarizations used in pulse transmission. Although $\left\langle\left|V_{\theta}\right|^{2}\right\rangle_{m}$ and $\left\langle\left|V_{\mathrm{o}}\right|^{2}\right\rangle_{m}$ will individually depend on the polarization of transmitted field and magneto-ionic propagation effects, the sum $\left\langle\left|V_{\theta}\right|^{2}\right\rangle_{m}+\left\langle\left|V_{\mathrm{o}}\right|^{2}\right\rangle_{m}$ is independent of these factors since the orthogonal field samples $V_{\theta}$ and $V_{\circ}$ fully account for the scattered field and its entire power content whatever the polarization.

[13] Transmitter pulses used in Jicamarca drifts experiments have a 3-baud Barker code (++-) with a baud length of $\Delta z=15$. Hence, to account for the power data obtained with Barker decoded signal samples we use in (4) the ideal data model

$$
\left\langle\left|V_{\theta}\right|^{2}\right\rangle_{m}+\left\langle\left|V_{\mathrm{o}}\right|^{2}\right\rangle_{m}=\Pi_{m-2}+9 \Pi_{m}+\Pi_{m+2}
$$

which is a straightforward extension of (7).

\subsubsection{Differential-Phase Model}

[14] For transverse-propagating and linear-polarized radar returns (1) implies that

$$
\left\langle\left\langle V_{\theta} V_{\odot}^{*}\right\rangle_{m} \approx-\frac{80.6}{c f^{2} \omega} \sum_{q=1}^{m} \Omega_{q}^{2} N_{q} \Delta z \equiv \Psi_{m},\right.
$$

where $\Omega_{q} \equiv \Omega\left(z_{q}\right)$, product $N_{1} \Delta z$ represents the total electron content of lower ionosphere below the lowest usable data range gate $z_{1}$, and, for $m>1, N_{m}$ is the average electron density between $z_{m-1}$ and $z_{m+1}$. However, when the effect of elliptic-polarized radar returns propagating in slightly offtransverse directions is taken into account, a small correction term $\delta \Psi_{m}$ needs to be added to $\Psi_{m}$. Therefore, in (5) we use

$$
\left\langle\left\langle V_{\theta} V_{o}^{*}\right\rangle_{m}=\Psi_{m}+\delta \Psi_{m},\right.
$$

where the correction term $\delta \Psi_{m}$ is determined by considering the axial-ratio and differential-phase of backscattered returns propagating at an angle $0.38^{\circ}$ away from the transverse 
direction as explained in detail in a forthcoming paper by $\mathrm{Z}$. Feng et al. (Transverse-beam incoherent scatter radar measurements of F-region plasma densities at Jicamarca, submitted to Radio Science, 2002, hereinafter referred to as Feng et al., submitted manuscript, 2002). The angle $0.38^{\circ}$ used in $\delta \Psi_{m}$ calculations is the $\sigma$ of a Gaussian curve that describes the shape of the two-way antenna gain pattern at Jicamarca after it has been integrated in zonal direction perpendicular to $\vec{B}$.

\subsubsection{Data/Model Misfit}

[15] From the viewpoint of least-squares data fitting (6) represents a global misfit between the data and data models described above. The computation and minimization of the misfit requires that its summation bounds over $m$ be specified and data variances $\left\langle\delta P_{m}^{2}\right\rangle,\left\langle\delta \psi_{m}^{2}\right\rangle$ known. The summation bounds in turn depend on how accurately the variances can be specified.

[16] Let $s_{m}$ denote the normalized cross-correlation of $\theta$ and $\phi$-channel outputs at range gate $z_{m}$ and $I$ the number of independent voltage products averaged to obtain the differential phase estimate $\psi_{m}$. Then, as shown in Woodman and Hagfors [1969], error $\delta \psi_{m}$ in $\psi_{m}$ is a Gaussian random variable with a variance

$$
\left\langle\delta \psi_{m}^{2}\right\rangle=\frac{1}{2 I} \frac{1-\left|s_{m}\right|^{2}}{\left|s_{m}\right|^{2}}
$$

provided that

$$
\left|s_{m}\right| \gg \frac{1}{\sqrt{I}}
$$

Condition (12) can be satisfied only near and below the F-region peak because above the F-region peak $\left|s_{m}\right|$ decreases with increasing altitude as the signal-to-noise ratio (SNR) drops. For that reason the second sum in (6) is carried from $m=1$ corresponding to the first data range gate $z_{1}=210 \mathrm{~km}$ to some $m$ above the F-region peak above which (12) is violated. The first sum in (6), on the other hand, is carried from $z_{m}=300$ to $825 \mathrm{~km}$ using the variance formula

$$
\left\langle\delta P_{m}^{2}\right\rangle=\frac{\left\langle P_{m}\right\rangle^{2}}{I}-\left(1-\left|s_{m}\right|^{2}\right) \frac{2|g|^{2}\left\langle P_{\theta m}\right\rangle\left\langle P_{\mathrm{o} m}\right\rangle}{I} .
$$

Power data for $z_{m}<300 \mathrm{~km}$ are excluded from (6) for a number of reasons including the dependence of $(8)$ on unknowns $\mu_{m}$.

[17] In computing $\left\langle\delta \psi_{m}^{2}\right\rangle$ and $\left\langle\delta P_{m}^{2}\right\rangle$ according to (11) and (13) we replace $\left|s_{m}\right|$ and $\left\langle P_{m}\right\rangle,\left\langle P_{\theta, o m}\right\rangle$ with their estimates obtained from the measured data. Also the value for $I$ is determined from the time integration used to compute $P_{\theta, \mathrm{om}}$, $\psi_{m}$.

\subsection{Data Inversion}

[18] In section 2.2 we found out that given a set of radar data $P_{\mathrm{\theta}, \mathrm{o}}, \psi_{m}$, misfit (6) is a function of ionospheric density samples $N_{m}$ as well as parameters $K,|g| \angle g, A_{\theta, o}$, and $\mu_{m}$. We also know that $\mu_{m}=1$ for $z_{m} \geq 450 \mathrm{~km}$ (the altitude regime where $T_{e} / T_{i} \approx 1$ is expected) and we have fairly good estimates for the noise power $A_{\theta, \circ}$ obtained from the highest sampled range gates $\left(z_{m}=840\right.$ to $\left.900 \mathrm{~km}\right)$ which are excluded from the $\chi^{2}$ sum. Furthermore, we expect that $|g| \approx 1$ because the receiver gains were fairly well balanced during the experiment. Therefore, the best fit of the measured data to the models described in section 2.2 is obtained by minimizing

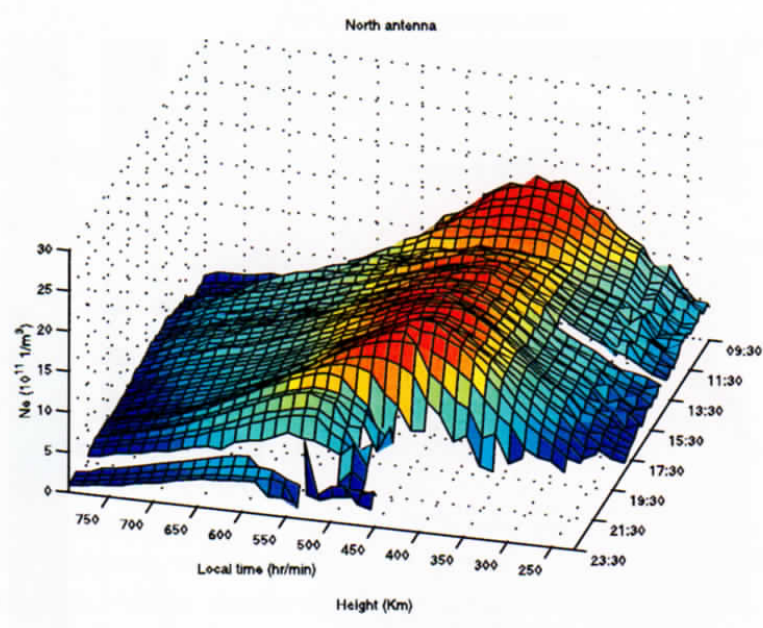

Figure 1. Surface plot of estimated plasma density $N$ for January 9, 2000.

(6) over $K,|g|, \angle g, A_{\theta, \mathrm{o}}, \mu_{m}$ (for $300<z_{m} \leq 450 \mathrm{~km}$ ), and $N_{m}$ subject to the constraints that (a) $A_{\theta, o}$ remain within a standard deviation of their independently determined estimates, and (b) $|g|$ remains close to 1 . The constraints are enforced by adding to (6) terms which grow quadratically when $A_{\theta, o}$ and $|g|$ stray away from their expected values ("prior information" within a Bayesian framework). However, deviation of $|g|$ from 1 is not heavily penalized.

[19] The fit or data inversion procedure outlined above is applied to power and phase data $P_{\theta, o m}, \psi_{m}$ obtained with 15 minutes of time integration. The inversion procedure is initiated with reasonable guesses for the unknowns which are subsequently adjusted iteratively using the MarquardtLevenberg algorithm [e.g., Press et al., 1988] until the modified $\chi^{2}$ is minimized to a value on the order of the number of degrees of freedom of the problem. The convergence time per altitude profile is less than $10 \mathrm{~s}$ with a MATLAB code on a PC, i.e., a small fraction of an integration time, opening the possibility for real-time evaluations. System unknowns $K,|g|, \angle g$, as well as noise powers $A_{\theta, \mathrm{o}}$ change very little from one profile inversion to the next.

[20] Before discussing the inversion examples shown in Figures 1 and 2 we need to describe a few more aspects of the inversion process. First, we note that it is not possible to lump the unknown $\angle g$ with the input $N_{1}$ of $\Psi_{m}+\delta \Psi_{m}$ model because of the nonlinear dependence of $\delta \Psi_{m}$ on $N_{1}$ described in Feng et al. (submitted manuscript, 2002). Thus the receiver phase $\angle g$ remains an independent free parameter of our inversion process. This does not cause a major difficulty as we find that the best fit values of $\mathrm{Lg}$ remain unchanged between profile inversions.

[21] Second, we note that the success of our density profile inversions depends crucially on having both power and phase data $P_{\theta, o m}$ and $\nu_{m}$ at a sufficiently large number of range gates above $450 \mathrm{~km}$ where $\mu_{m}=1$ is assumed. The mutual consistency of $P_{\theta, o m}$ and $\psi_{m}$ variations over those range gates is effectively what determines the values of $K, g$, and $A_{\theta, o}$, and thus make density estimation possible in the lower and upper F-regions where either power or phase data are unreliable and/or not useful. Density estimates from upper F-region, for instance, only depend on power data combined with $K,|g|$, and $A_{\theta, \mathrm{o}}$ coefficients since phase data are unreliable (and excluded from (6)) because of the 


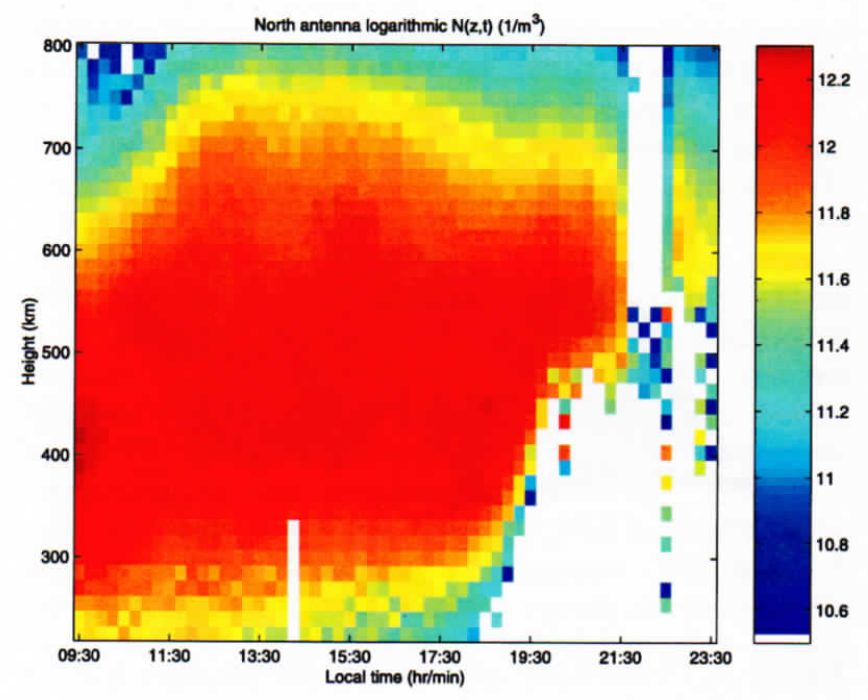

Figure 2. Map plot of $\log _{10} N$ estimates for January 9, 2000. Missing data in the evening sector are a consequence of receiver saturation due to spread-F echoes.

violation of (12). Conversely, for density estimates from lower F-region where $\mu_{m}$ is a free parameter (instead of unity) only the phase data matter. The reason is, whatever the power data, an unconstrained $\mu_{m}$ always adjusts itself during the inversion to cancel out $\delta P_{m}$ exactly within (6). Therefore, power data $P_{\theta, \mathrm{om}}$ are superfluous for density estimation in the lower F-region unless $\mu_{m}$ is constrained based on some prior knowledge.

[22] It should be clear from above discussion that random fluctuations in lower F-region density estimates originating from phase errors $\delta \psi_{m}$ can be reduced or regularized [e.g., Press et al., 1988] by preventing $\mu_{m}$ from fluctuating freely. One way of doing that which was used in the examples shown in Figures 1 and 2 is to penalize the deviations of $\mu_{m}$ from its neighbor $\mu_{m+1}$. This is a reasonable constraint on $\mu_{m}$ since $T_{e} / T_{i}$ is known to vary slowly with height. The constraint is enforced by adding a term $\alpha \Sigma_{m}\left(\mu_{m}-\mu_{m+1}\right)^{2}$ to (6) before minimization. The sum in this new term is carried over $300<z_{m} \leq 450 \mathrm{~km}$ altitude region and the penalty coefficient $\alpha$ is taken (more or less) as the smallest value for which density noise in the same region is substantially reduced. Constrained as well as unconstrained (i.e., $\alpha=$ 0 ) inversions typically yield $\mu_{m}$ values of 0.3 to 1.2 between 300 and $450 \mathrm{~km}$ altitudes. Also, as expected, constrained inversions result in smoother variations of $\mu_{m}$ and $N_{m}$ with height. In the next section we will explain why $\mu_{m}<1$ is reasonable when $T_{e} / T_{i}>1$ at lower F-region heights.

\section{Discussion}

[23] Figures 1 and 2 display F-region plasma density estimates $N_{m}, m>1$, as a function of sampling heights $z_{m} \equiv$ $210+(m-1) 15 \mathrm{~km}$ obtained with the data inversion procedure described in section 2 . The radar data used in the inversions were collected at the Jicamarca Radio Observatory on January 9, 2000, using the transverse beam geometry of the Jicamarca F-region drifts experiments and an interpulse period (IPP) of $6.6 \mathrm{~ms}$. The transmitted radar pulses were coded using the ++- Barker code with a baud length of $15 \mathrm{~km}$ and the return signals were matched filtered in the baseband. Missing density data in Figures 1 and 2 in the evening sector are a consequence of receiver saturation due to equatorial spread-F echoes.

[24] The results presented Figures 1 and 2 should be regarded as preliminary as we are aware that the inversions can be improved by taking into account minor sources of systematic errors (e.g., I- and Q-channel misbalances, instrumental coupling between the $\theta$ - and $\phi$-channels, small deviation of beam boresights from the transverse direction, etc.) as well as including in the procedure the Jicamarca ionosonde data. Also, with the wide dynamic range digital receivers being developed at Jicamarca it will be possible to detect the F-region returns without saturation problems even during spread-F conditions. Since phase noise will be substantially reduced during spread-F due to increased SNR we expect that it will be possible to make density measurements within equatorial spread-F layers and bubbles using the differential-phase data. Note that the height derivative of the differential-phase of spread-F echoes can be modeled in terms of linear polarized ordinary- andextraordinary modes - just like (1) - since spread F irregularities are known to be highly field aligned and the variation of $\vec{B}$ within a single range gate is negligible.

[25] In our density estimation procedure we treat $\mu$ as a regularized unknown in the lower F-region since the latter depends on $T_{e} / T_{i}$ which can deviate from 1 at those altitudes. The dependence is $\mu \approx T_{e} / T_{i}$ for longitudinal modes, but for transverse modes, $\mu \approx T_{i} / T_{e}$. Lower $\mathrm{F}$ region $\mu_{m}$ estimates which were found to be less than 1 in our inversions indicate that the backscattered fields were dominated by tranverse propagating contributions as expected.

[26] Acknowledgments. We appreciate inspired inputs and suggestions from Koki Chau and Farzad Kamalabadi and thank the staff and engineers of the Jicamarca Radio Observatory for their assistance with the observations. The Observatory is operated by Instituto Geofisico del Peru with support from the U. S. National Science Foundation. This work was supported by the Aeronomy Program, Division of Atmospheric Sciences of the NSF through grants ATM 99-11209 and ATM 02-15426.

\section{References}

Farley, D. T., Faraday rotation measurements using incoherent scatter, Radio Sci., 4, 143, 1969.

Farley, D. T., Radio wave scattering from the ionosphere, in Methods of Experimental Physics, vol. 9, part B, edited by R. H. Lovberg and H. R. Griem, Academic, San Diego, Calif., 1971.

Kudeki, E., et al., A new approach in incoherent scatter $F$ region $\mathrm{E} \times \mathrm{B}$ drift measurements at Jicamarca, J. Geophys. Res., 104, 28,145, 1999.

Menke, W., Geophysical Data Analysis: Discrete Inverse Theory, Academic, San Diego, 1989

Press, W. H., et al., Numerical Recipes in C, Cambridge Univ. Press, New York, 1988.

Ratcliffe, J. A., The Magneto-ionic Theory, Cambridge Univ. Press, New York, 1962.

Valladares, C. E., and R. F. Woodman, Comparison of Jicamarca densities and a numerical first-principle model of the low latitude ionosphere, paper presented at CEDAR meeting, Longmont, Colo., 2001.

Woodman, R. F., and T. Hagfors, Methods for the measurement of vertical ionospheric motions near the magnetic equator by incoherent scattering, J. Geophys. Res., 74, 1205, 1969.

Woodman, R. F., et al., Synthesis of Jicamarca data during the great storm of March 8, 1970, Radio Sci., 7, 739, 1972.

Z. Feng and E. Kudeki, Department of Electrical and Computer Engineering, University of Illinois, 1406 W. Green Street, Urbana, IL 61801, USA. (e-kudeki@uiuc.edu)

R. F. Woodman, Instituto Geofisico del Peru, Lima, Peru. (ronw@ geo.igp.gob.pe) 